\title{
Las fundaciones universitarias andaluzas como sujetos de la contratación pública
}

\author{
Manuel Mesa Vila \\ Abogado especialista en contratación pública \\ Letrado asesor de fundaciones del sector público
}

\begin{abstract}
Las fundaciones creadas por las universidades son un fenómeno muy extendido en el ámbito territorial de Andalucía, donde todas las universidades públicas cuentan con al menos una fundación que actúa como entidad instrumental.
\end{abstract}

El presente artículo analiza dos aspectos relacionados con el régimen jurídico de contratación de las fundaciones universitarias: el grado de sometimiento de este tipo de entidades a la Ley 30/2007, de Contratos del Sector Público, y las relaciones instrumentales con la universidad creadora, asunto de especial trascendencia y que se encuentra condicionado al cumplimiento de unos límites y requisitos establecidos en la ley y que han sido objeto de una prolija interpretación en la jurisprudencia europea.

\section{INTRODUCGIÓN}

La utilización de la forma jurídica de fundaciones como entidades instrumentales ha proliferado en los últimos años en el seno de las administraciones públicas. Estado, comunidades autónomas y entidades que integran la administración local, han visto en la fundación el modelo idóneo para la gestión de determinadas parcelas de su acción pública.

No en vano, las fundaciones tienen la ventaja de contar en su definición con elementos que coinciden con la esencia de la Administración Pública: ya que son organizaciones constituidas sin ánimo de lucro que tienen afectado de modo duradero su patrimonio a la realización de fines de interés general. Así pues, se trataría de una forma jurídica mucho más apropiada para la presta- 
ción de determinados servicios y el cumplimiento de determinados fines que las sociedades mercantiles. La propia exposición de motivos de la Ley de Fundaciones de Andalucía considera a éstas, sean públicas o privadas, como "colaboradoras de los poderes públicos en el ejercicio de sus actividades de interés general".

Esta proliferación no ha estado exenta de críticas de todas partes. Por un lado la doctrina administrativista que ha contemplado en este fenómeno una nueva huida del derecho administrativo, y por otro, la doctrina iusprivatista que considera que el uso instrumental de las fundaciones supone una desnaturalización de la institución fundacional, basada en un patrimonio que por voluntad de su creador se afecta de modo duradero a la realización de fines de interés general; afirman que en las fundaciones creadas por la administración predomina el aspecto instrumental, en principio secundario, sobre el elemento esencial, que es el patrimonio.

Según los datos recogidos en el Informe Económico-Financiero del Sector Público Empresarial y Fundaciones referenciado a 2006 de la Intervención General de la Administración General del Estado, en España existen un total de 323 fundaciones del sector público de las comunidades autónomas, Andalucía participa de ese total con 19 fundaciones, que suponen tan solo un $6 \%$ del total nacional.

Para determinar el número de fundaciones del sector público estatal hemos de recurrir a la Ley Presupuestos Generales del Estado, en la que anualmente aparece la relación de fundaciones estatales, son en la actualidad un total de 42. Las fundaciones municipales, creadas por ayuntamientos, diputaciones y mancomunidades, suponen otra importante aportación a este extenso panorama.

Esta proliferación y los problemas jurídicos que suponen la gestión de este tipo de entidades de derecho privado que actúan en el ámbito de lo público ha obligado a cada una de las administraciones públicas a sentar unas bases mínimas que confieren un estatus jurídico especial a las fundaciones que de ellas dependen, sin que podamos decir lo mismo de las fundaciones universitarias.

La universidad como ente de derecho público prestador del servicio de educación superior, no ha sido ajeno a este fenómeno, que cuenta con una habilitación legal expresa recogida en el Artículo 84 de la Ley 6/2001, Orgánica de Universidades (LOU): 
Para la promoción y desarrollo de sus fines, las Universidades, con la aprobación del Consejo Social, podrán crear, por sí solas o en colaboración con otras entidades públicas o privadas, empresas, fundaciones u otras personas jurídicas de acuerdo con la legislación general aplicable.

Por su parte, el artículo 83 de la Ley 6/2001, Orgánica de Universidades (LOU) establece:

Los grupos de investigación reconocidos por la Universidad, los Departamentos y los Institutos Universitarios de Investigación, y su profesorado a través de los mismos o de los órganos, centros, fundaciones o estructuras organizativas similares de la Universidad dedicados a la canalización de las iniciativas investigadoras del profesorado y a la transferencia de los resultados de la investigación, podrán celebrar contratos con personas, Universidades o entidades públicas y privadas para la realización de trabajos de carácter científico, técnico o artístico, así como para el desarrollo de enseñanzas de especialización o actividades específicas de formación.

El artículo reproducido marca pues, el objeto y finalidad que, en principio debe mover la creación de fundaciones por parte de las universidades:

- La canalización de iniciativas investigadoras del profesorado.

- La transferencia de los resultados de la investigación

- El desarrollo de enseñanzas de especialización o actividades específicas de formación.

Por tanto, el objeto y finalidad de las fundaciones universitarias no es, ni puede ser en ningún momento, la gestión del servicio público de educación superior, servicio que debe realizar directamente la Universidad mediante la investigación, la docencia y el estudio (artículo 1, LOU). La finalidad de estas entidades debe ir encaminada tanto al fomento de puntos de encuentro entre la Universidad y su entorno cultural, profesional, económico y social (de ahí la participación del Consejo Social en su creación) como al desarrollo de actividades dirigidas de forma genérica al fomento de la investigación, la docencia, la cultura y la educación.

El establecimiento de cauces de diálogo entre la Universidad y la sociedad mediante la promoción de programas de estudio, investigación o docencia que vayan dirigidos a dar respuesta a las necesidades socioeconómicas y culturales de ésta, propiciando para ello la formación continuada, el reciclaje tecnológico y la promoción de la investigación, o mediante la creación de bolsas de trabajo destinadas a la orientación o la inserción profesional de los estudiantes, 
son algunos de los objetivos que pretenden las fundaciones constituidas por las universidades públicas que existen en la actualidad.

Pero además de estos nobles objetivos materiales, siempre subyace un objetivo formal que no es otro que incrementar la eficacia y la eficiencia de las organizaciones huyendo de la rigidez del derecho administrativo hacia formas de gestión privadas, por naturaleza más eficaces y adaptables que el derecho público. Esta es el trasfondo que subyace en el artículo 83 de la LOU.

Sabedoras las universidades de la rigidez que les da a sus estructuras el ejercicio de las potestades implícitas en el servicio público en entidades de base administrativa y la existencia de personal funcionario para desempeñar las tareas de esta índole, se acudió a personificar, particularmente en fundaciones, estas actividades, lejos de la hiperinflación universitaria de representatividad en todos sus órganos colegiados, y buscando organizaciones más eficientes para estos empeños. Porque la universidad conocía que el camino de las fundaciones resultaba útil para relacionarse con el sector privado, ya que así se lo había demostrado la organización de trabajos de investigación, o de menor pretensión científica, pero técnica al cabo, sabrosos para las economías individuales de los profesores, al ser frecuentados por los empresarios para recabar la ayuda de la universidad, por los cauces de la LRU, que permitían incrementos del siempre corto sueldo docente. Y también, todo hay que decirlo, porque los controles efectuados por el protectorado a las universidades eran menos exigentes que los que podría organizar el Tribunal de Cuentas estatal o las diferentes cámaras de cuentas de las comunidades autónomas. Que no eran los peores, pues nada es más terrible que las envidias cainitas de un claustro universitario. ${ }^{1}$

La fórmula, como indica Juan Manuel del Valle ${ }^{2}$, ha brindado servicios innegables a la sociedad y a la comunidad universitaria, y ha sido utilizada a similares fines. Su imagen, además, responde a una semiología de filantropía social cara para los universitarios, ajena a otros significantes de carácter mercantil o civil, que pudieran dar una falsa imagen de contaminación por la vida cotidiana. Tampoco es ajena al fenómeno, en absoluto, una antigua vinculación de fundaciones y Universidades, al crear mediante aquéllas estructuras de éstas.

${ }^{1}$ Por Juan Manuel del Valle Pascual. Las fundaciones universitarias como instrumentos para una politica activa de empleo. Actualidad Administrativa, No 15, Sección A Fondo, Quincena del 1 al 15 Sep. 2005, pág. 1797, tomo 2, Editorial LA LEY.

2 En Las Fundaciones Universitarias. Diario La Ley, Sección Doctrina, 1999, Ref. D-307, tomo 6, Editorial LA LEY. 


\subsection{Configuración Jurídica de las Fundaciones Universita- rias}

El debate sobre la naturaleza jurídica de las universidades, a pesar del tiempo transcurrido desde sus orígenes, continúa abierto. Muchos han sido los autores que han reflexionado sobre el tema y muchas las opiniones. En el presente análisis no vamos a entrar en ese asunto, pero debemos tomar partido sobre la naturaleza jurídica de las universidades públicas como entes promotores y fundadores, si queremos definir y delimitar la naturaleza de las mismas. Del mismo modo que para identificar a un hijo es necesario partir de la identificar quienes son sus padres, trataremos de situarnos en la tesis más extendida.

La doctrina científica, respecto al problema de la naturaleza jurídica de las universidades, es tan extensa que en un extremo podríamos considerar a los autores que continúan admitiendo su naturaleza de organismos autónomos, pasando por aquellos otros que hablan de la «naturaleza institucional», siguiendo los que afirman se trata de un «establecimiento público personificado», aquellos que consideran se trata de una auténtica «corporación», los de más allá que las estiman como «entes especializados con autonomía real», haciendo otros especial referencia a las Universidades como «entidades públicas fundacionales», llegando a los que las consideran «entes públicos de naturaleza institucional de carácter especial» ${ }^{3}$

Lo que si parece claro e indiscutible es su condición de ente de derecho público, dado que su cometido primordial es la prestación de un servicio público (servicio público de la educación superior), y por tanto, su consideración de ente jurídico-público, en línea con el empeño tan necesario en derecho de dividir las entidades en dos: las públicas y las privadas.

En esa consideración de ente jurídico público situamos el nacimiento de las fundaciones universitarias, ya que las vigentes leyes de fundaciones, en desarrollo del derecho de fundación reconocido sin matices de ningún tipo en el artículo 34 de la Constitución Española, reconoce el derecho de fundación haciendo esa bipolarización tan recurrente en derecho de las personas jurídico privadas y las jurídico públicas.

\footnotetext{
3 "La Administración universitaria: algunos aspectos sobre legalidad y realidad". Ana Isabel Caro Muñoz y Pilar Peña Callejas, Manuel Souto Alonso y Manuel Marrón Serrano. Actualidad Administrativa, Sección Doctrina, 2001, Ref. VIII, pág. 183, tomo 1, Editorial LA LEY.
} 


\section{Artículo 8. Capacidad para fundar.}

1. Podrán constituir fundaciones las personas fisicas y las personas jurídicas, sean éstas públicas o privadas.

4. Las personas jurídico-públicas tendrán capacidad para constituir fundaciones, salvo que sus normas reguladoras establezcan lo contrario.

Exactamente en los mismos términos se expresa el artículo 8 de la ley 10/2005, de Fundaciones de la Comunidad Autónoma de Andalucía.

Ambos declaran la plena capacidad de las personas jurídico públicas para constituir fundaciones (asunto éste que tampoco es pacífico y que también es objeto de un amplio debate doctrinal) estableciendo como única limitación que sus normas reguladoras establezcan lo contrario.

Y su norma básica reguladora, no sólo no establece lo contrario, sino que admiten expresamente tal posibilidad:

Para la promoción y desarrollo de sus fines, las Universidades, con la aprobación del Consejo Social, podrán crear, por sí solas o en colaboración con otras entidades públicas o privadas, empresas, fundaciones u otras personas jurídicas de acuerdo con la legislación general aplicable (Artículo 84 de la Ley 6/2001, Orgánica de Universidades).

Este expreso reconocimiento de la posibilidad que tienen las universidades de crear fundaciones, se ve aun más reforzado por el principio de autonomía universitaria proclamado por el artículo 27 de la Constitución Española.

La legislación andaluza, no vuelve en este caso, como ocurría en la delimitación de la capacidad para fundar, a proclamar la posibilidad de crear fundaciones sino que admite de forma implícita lo dispuesto en el artículo 84 de la LOU dando por sentada la vigencia de tal posibilidad en el ámbito de las universidades públicas andaluzas y estableciendo algunas formalidades específicas relativas a la creación y funcionamiento.

Por tanto, nos encontramos ante fundaciones que cuentan con su correspondiente previsión legal, tanto en la ley de fundaciones como en las leyes básicas reguladoras de las universidades, que son creadas por personas jurídico públicas o de derecho público. Esa naturaleza jurídico - pública del fundador es lo que determina que en una primera lectura podamos afirmar que nos encontramos ante fundaciones públicas, sin que esta afirmación en principio ten- 
ga otra implicación y utilidad que la de diferenciarlas de las fundaciones privadas, que serían aquellas cuyo fundador es un persona física o una entidad privada.

No obstante, afirmar que las fundaciones universitarias responden a la categoría de fundaciones públicas, no otorga a este tipo de entidades un estatus jurídico especial, sino que habrá que atender a cada una de las facetas de su gestión, como la gestión de personal, el régimen de contratación y compras, el régimen económico, etc. para responder si tienen o no un estatuto jurídico especial.

Las tipologías de fundaciones públicas con expreso reconocimiento en el ordenamiento jurídico son las siguientes:

- Las Fundaciones de sector público estatal, reguladas en el capítulo XI de la Ley 50/2002, de Fundaciones.

- Fundaciones del sector público andaluz, reguladas en la Ley 10/2005, de Fundaciones de la Comunidad Autónoma de Andalucía, cuyo capítulo $\mathrm{X}$ define y regula este tipo de entidades, contando además con la remisión legal expresa de una norma de derecho administrativo cual es la Ley 9/2007, de la Administración de la Junta de Andalucía.

- Fundaciones públicas locales, han sido las últimas en contar con un estatuto jurídico especial establecido por una ley, en este caso se trata de la Ley 5/2010, de 11 de junio, de Autonomía Local de Andalucía.

Estos tres tipos de fundaciones comparten un régimen jurídico similar, en los tres casos se repite la prohibición de ejercicio de potestades públicas, como consecuencia lógica de su carácter de entidades de derecho privado. En el ámbito que nos interesa de la contratación, las tres deben someterse a lo que disponga la normativa básica estatal en la materia y a la que corresponda aprobar a la Comunidad Autónoma. Siendo ese el análisis en que se centran los siguientes apartados.

Otro tipo de fundaciones públicas sui generis son las fundaciones públicas del servicio, las fundaciones públicas sanitarias y las fundaciones del patrimonio nacional.

Las Fundaciones Públicas del Servicio fueron creadas por el artículo 67.2 ${ }^{\mathrm{a}}$ del Reglamento de Servicios de las Corporaciones Locales, de 17 de junio de 
1955, como una de las modalidades de gestión directa de los servicios municipales. Encuentran su regulación en los artículos 85 a 88 del mismo reglamento. Este tipo de entidades forman parte de la Administración Institucional, su régimen jurídico es de derecho público. Una parte de la doctrina considera que este término se encuentra reemplazado por el de "organismo autónomo local" que es el considerado por el artículo 85.3.b) de la Ley Reguladora de las Bases de Régimen Local, de 2 de abril de 1985. Se trata en definitiva de auténticos organismo autónomos locales sometidos a derecho administrativo.

La denominación de fundación pública sanitaria aparece en la Ley 15/1997, de 25 de abril, sobre habilitación de Nuevas formas de Gestión del Sistema Nacional de Salud y su régimen jurídico se desarrolla en el Real Decreto 29/2000, de 14 de enero. A pesar de su nombre, en estos casos no nos encontramos ante auténticas fundaciones sino ante entidades de derecho público con personalidad jurídico - pública creadas por la Administración. Son organismos públicos adscritos al Instituto Nacional de Salud, que se rigen subsidiariamente por lo dispuesto para las entidades públicas empresariales en la LOFAGE. La Disposición Adicional Tercera de la Ley 50/2002, de Fundaciones, excluye a estas fundaciones de su ámbito de aplicación.

Por último, las Fundaciones del Patrimonio Nacional, constituyen una exclusión expresa recogida en la Disposición Adicional Primera de la Ley 50/2002, de Fundaciones. En virtud de esta exclusión la ley no será de aplicación a las fundaciones a que se refiere la Ley 23/1982, del Patrimonio Nacional. Según la citada ley, forman parte del Patrimonio Nacional los derechos de patronato o de Gobierno y administración sobre una serie de Fundaciones, denominadas Reales Patronatos.

\section{¿Dónde encuadramos a las fundaciones universitarias?}

Como afirmación categórica en este punto podemos afirmar que las fundaciones universitarias no pueden ser encuadradas en ninguna de las categorías anteriores.

La que más duda podría plantear es la posibilidad de considerarlas fundaciones del sector público andaluz en una interpretación muy estricta de la ley: si contemplamos una de las posibilidades que brinda el artículo 55 se consideran fundaciones del sector público de la Comunidad Autónoma de Andalucía aquellas que se constituyan con una aportación mayoritaria, directa o indirecta, de la Administración de la Junta de Andalucía, sus organismos públicos o demás entidades o empresas de la Funta de Andalucía. Si tenemos en cuenta el modelo de financiación de las universidades 
públicas basado en la transferencia de fondos públicos procedentes de la Junta de Andalucía, podríamos en principio considerar que existe una aportación mayoritaria indirecta en la constitución de las fundaciones universitarias, pero esto podría ser una visión muy simplista del fenómeno y entraría en conflicto con la interpretación teleológica de la norma y con el principio de autonomía universitaria, que nos lleva a descartar por completo esta posibilidad.

Por tanto, las fundaciones universitarias, son fundaciones públicas en virtud del carácter público de su fundador, sin que en principio ese apellido tenga ninguna trascendencia jurídica per se, sino como simple contraposición de las fundaciones privadas.

Tendrían que haber sido las leyes sustantivas reguladoras de las universidades (Ley 6/2001, Orgánica de Universidades y Ley 15/2003, de 22 de diciembre, Andaluza de Universidades), el marco idóneo para establecer el régimen jurídico peculiar de las fundaciones universitarias, cosa que no ha ocurrido limitándose las citadas normas a declarar la posibilidad de crearlas sometiéndola a las autorizaciones y requisitos establecidos para su creación y para su financiación.

\subsection{Régimen Jurídico Básico}

Como hemos indicado la posibilidad de crear entidades jurídicas diferenciadas que contribuyan a la consecución de sus objetivos, es una capacidad reconocida a las universidades españolas en la LOU, que se limita a reconocer tal posibilidad ejercida por si mismas o en colaboración con otras entidades públicas o privadas, incluyendo tan sólo dos normas imperativas al respecto:

La primera es la necesidad de la previa aprobación del Consejo Social. La intervención de este órgano de participación, como hemos indicado, deviene lógica cuando la finalidad de estas entidades debe ser el fomento de puntos de encuentro entre la Universidad y su entorno cultural, profesional, económico y social.

La segunda, recogida en el segundo párrafo del artículo 84, establece el sometimiento de las fundaciones y el resto de entidades en que las Universidades tengan participación mayoritaria, a la obligación de rendir cuentas en los mismos plazos y procedimiento que las propias Universidades.

El legislador estatal deja en manos de las comunidades autónomas el desarrollo de la regulación específica de este tipo de entidades, incluyendo ex- 
presamente el régimen de la dotación fundacional o la aportación al capital social y cualesquiera otras aportaciones a las entidades que se creen, con cargo a los presupuestos de la Universidad.

El legislador andaluz asume el mandato establecido en la LOU, aprobando la Ley 15/2003, de 22 de diciembre, Andaluza de Universidades que regula determinados aspectos relativos a las fundaciones universitarias:

En primer lugar, reconoce en el artículo 11 que la creación, modificación y supresión de departamentos y de cualesquiera estructuras específicas que actúen como soporte de la investigación y de la docencia (entre ellas las fundaciones) corresponde exclusivamente a cada Universidad conforme a sus Estatutos o a sus normas de organización y funcionamiento.

La previsión del artículo 11 supone la aplicación del principio de autonomía universitaria que da soporte a todo el sistema. En el momento de la creación de la fundación interviene única y exclusivamente la voluntad de la propia universidad expresada a través de sus órganos de gobierno. El único trámite externo obligatorio que establece la Ley 15/2003, en su artículo 96.4 es la comunicación a la Consejería de Economía y Hacienda, dentro de los 15 días siguientes a aquél en que se adopte el acuerdo de creación o participación. Se trata de una comunicación con meros efectos informativos sin que en ningún caso tenga el carácter de informe vinculante, y sin que en principio la Consejería pueda alegar ni oponer ningún argumento en contra de la creación de la entidad.

En segundo lugar, el artículo 18 reitera la previsión del artículo 84 de la LOU citando entre las funciones del Consejo Social la aprobación de las fundaciones u otras entidades jurídicas que las Universidades, en cumplimiento de sus fines, puedan crear por sí solas o en colaboración con otras entidades públicas o privadas.

En tercer lugar, el artículo 96, es el que contiene un mayor número de normas imperativas, en este artículo se regula, tal y como preveía el 84 de la LOU, el régimen de la dotación fundacional o la aportación al capital social y cualesquiera otras aportaciones de la universidades a sus fundaciones:

1. La dotación fundacional o aportación al capital social de entidades que las Universidades creen al amparo del artículo 84 de la Ley Orgánica de Universidades estará sometida a los siguientes criterios: 
a) Tendrá asignada dotación específica en los presupuestos de la Universidad.

b) Será proporcionada a la viabilidad estimada de la consecución de los objetivos académicos, sociales y económicos de la entidad.

c) No podrán aportarse bienes de dominio público universitario más que en régimen de concesión o cesión de uso, estableciéndose en el acuerdo fundacional su duración y retorno a la Universidad.

d) Se remitirá al Consejo Social para su aprobación el previo informe o memoria económica que justifique la idoneidad de la medida.

2. Las ampliaciones de las dotaciones fundacionales o aportaciones al capital social por parte de la Universidad estarán sometidas a los mismos requisitos indicados en el apartado anterior.

3. No tendrán la consideración de aportación al capital las subvenciones, transferencias corrientes, aportaciones de bienes o prestaciones de servicios académicos, de administración y gestión que se efectúen a fundaciones, asociaciones o sociedades mercantiles en virtud de convenios o contratos entre la Universidad y aquellas entidades que se creen en el futuro o que se hubieren creado con antelación a la presente Ley.

4. La creación de empresas, fundaciones o cualquier otro tipo de entidad, o la participación, en su caso, en el capital o fondo social de la misma deberá comunicarse a la Consejería de Economía y Hacienda, dentro de los 15 días siguientes a aquél en que se adopte el acuerdo de creación o participación.

5. Las empresas, fundaciones o cualquier otro tipo de entidad creada o participada por Universidades públicas andaluzas deberán elaborar un presupuesto de explotación y capital, que se integrará en el presupuesto de la propia Universidad a efectos de lo dispuesto en el artículo 93 de la presente Ley.

Asimismo, las entidades a que se refiere este artículo, en las que las Universidades tengan participación mayoritaria en su capital o fondo patrimonial equivalente, quedan sujetas a la obligación de rendir cuentas en los mismos plazos y procedimientos establecidos para las propias Universidades.

Por último, el artículo 94, que podría contemplarse como una vulneración del principio de autonomía universitaria, y que deriva directamente del artículo 81.3 de la LOU, establece que cada una de las operaciones de endeudamiento de las Universidades públicas andaluzas y las entidades dependien- 
tes de ellas requerirán la autorización de la Consejería de Economía y Hacienda. La verdadera novedad de esta previsión legal es la extensión de la obligación previa a las entidades dependientes, entre otras a las fundaciones universitarias.

\section{LAS FUNDACIONES UNIVERSITARIAS EN LA LEY DE CON- TRATOS DEL SECTOR PÚBLICO}

Las universidades públicas españolas aparecen incluidas dentro del ámbito subjetivo de aplicación de la Ley 30/2007, de 30 de octubre, de Contratos del Sector Público. En concreto el artículo 3.1.c. cita expresamente a las Universidades Públicas. El apartado 2 del mismo artículo, las considera a efectos del grado de aplicación de la ley, como Administración Pública, entrando, por tanto, de lleno en la categoría de poder adjudicador del apartado tercero.

Por su parte, el artículo 2.f considera dentro del ámbito de aplicación de la LCSP a

Las fundaciones que se constituyan con una aportación mayoritaria, directa o indirecta, de una o varias entidades integradas en el sector publico, o cuyo patrimonio fundacional, con un carácter de permanencia, este formado en más de un 50 por ciento por bienes o derechos aportados o cedidos por las referidas entidades

Por lo que, en principio, las fundaciones creadas y dotadas por las universidades, están dentro del ámbito de aplicación de la ley, teniendo además la consideración de poderes adjudicadores, ya que se trata de entidades que reúnen los requisitos acumulativos para ser considerados como tales establecidos en el artículo 3.3:

- Que tengan personalidad jurídica propia.

- Que hayan sido creados específicamente para satisfacer necesidades de interés general, las fundaciones por la propia naturaleza de la institución, tienen esa razón de ser.

- Que no tengan carácter industrial o mercantil, negación que también forma parte de la esencia de las fundaciones

- Que uno o varios sujetos que deban considerarse poder adjudicador de acuerdo con los criterios de este apartado 3 financien mayoritariamen- 
te su actividad, controlen su gestión, o nombren a mas de la mitad de los miembros de su órgano de administración, dirección o vigilancia

Por tanto, las relaciones jurídicas que pudieran establecerse entre la universidad creadora y su fundación, sería una relación entre dos entidades sujetas a la LCSP, en concreto, y a los solos efectos de la ley, entre una administración pública y un ente instrumental.

No obstante, el artículo 4.1.m de la LCSP establece como negocios o contratos excluidos del ámbito de aplicación de la ley

Los negocios juridicos en cuya virtud se encargue a una entidad que, conforme a lo señalado en el articulo 24.6, tenga atribuida la condición de medio propio y servicio técnico del mismo, la realización de una determinada prestación. No obstante, los contratos que deban celebrarse por las entidades que tengan la consideración de medio propio y servicio técnico para la realización de las prestaciones objeto del encargo quedaran sometidos a esta Ley, en los términos que sean procedentes de acuerdo con la naturaleza de la entidad que los celebre y el tipo y cuantía de los mismos, y, en todo caso, cuando se trate de contratos de obras, servicios o suministros cuyas cuantías superen los umbrales establecidos...

Se trata del reflejo en la legislación española de la teoría del "house providing" o medio propio que constituye una fuente inagotable de conflictos en el ámbito de la contratación pública europea y de continua alusión en la jurisprudencia del Tribunal de Justicia Europeo.

Para que esa relación jurídica que se pueda producir entre ambas entidades en el ámbito de la contratación quede amparada por la excepción del artículo 4.1.h) y, por tanto, excluida del ámbito objetivo de la ley se tienen que producir una serie de requisitos concurrentes descritos en el artículo 24.6:

A los efectos previstos en este articulo y en el articulo 4.1.n), los entes, organismos y entidades del sector publico podrán ser considerados medios propios y servicios técnicos de aquellos poderes adjudicadores para los que realicen la parte esencial de su actividad cuando estos ostenten sobre los mismos un control análogo al que pueden ejercer sobre sus propios servicios. Si se trata de sociedades, además, la totalidad de su capital tendrá que ser de titularidad publica.

En todo caso, se entenderá que los poderes adjudicadores ostentan sobre un ente, organismo o entidad un control análogo al que tienen sobre sus propios servicios si pueden conferirles encomiendas de gestión que sean de ejecución obligatoria para ellos de acuerdo con instrucciones fijadas unilateralmente por el encomendante y cuya retribución se fije por referencia a tarifas aprobadas por la entidad publica de la que dependan. 
La condición de medio propio y servicio técnico de las entidades que cumplan los criterios mencionados en este apartado deberá reconocerse expresamente por la norma que las cree o por sus estatutos, que deberán determinar las entidades respecto de las cuales tienen esta condición y precisar el régimen de las encomiendas que se les puedan conferir o las condiciones en que podrán adjudicárseles contratos, y determinará para ellas la imposibilidad de participar en licitaciones publicas convocadas por los poderes adjudicadores de los que sean medios propios, sin perjuicio de que, cuando no concurra ningún licitador, pueda encargárseles la ejecución de la prestación objeto de las mismas.

\section{LOS LÍMITES DEL MEDIO PROPIO INSTRUMENTAL APLICA- DO A LAS FUNDACIONES UNIVERSITARIAS}

Estamos en disposición de afirmar que, en principio, el exigente artículo 24.6 se cumple al cien por cien en las relaciones que se pudieran establecer entre las universidades y las fundaciones por ellas creadas. Pero veamos los requisitos concurrentes aplicados a las fundaciones uno por uno:

\section{$1^{\circ}$. Una determinada fundación universitaria podrá ser con- siderada medio propio y servicio técnico de la universi- dad cuando ésta ostente sobre aquélla un control análogo al que pueda ejercer sobre sus propios servicios}

El control análogo es un concepto jurídico indeterminado de profusa interpretación jurisprudencial en el ámbito europeo y supone el aspecto más controvertido y problemático de la teoría del "house providing", precisamente por la utilización de un término tan ambiguo e indeterminado. De ahí que no se pueda realizar una afirmación apriorística categórica de cuando nos encontramos ante esta situación en el ámbito de las fundaciones y sea necesario estudiar caso por caso.

Uno de los criterios más utilizados por la jurisprudencia para trazar la frontera del control análogo es la de la composición del capital o del fondo inicial de la entidad de que se trate, entendiendo que debe estar compuesto al cien por cien por capital público, de ahí que sistemáticamente niegue la cualidad de medio propio a las sociedades de economía mixta. Esa negación sistemática ha tenido incluso reflejo en la propia ley, que incluye una presunción iuris et de iure:

"Si se trata de sociedades, además, la totalidad de su capital tendrá que ser de titularidad publica". 
Con esta presunción el legislador entiende que una sociedad en que participe capital privado, por mínima que sea esa participación, jamás puede tener la consideración de medio propio respecto del poder adjudicador del que dependa porque jamás podrá darse la premisa de que exista un control análogo.

No obstante, la negación de lo uno no implica la afirmación de lo otro, es decir el supuesto de que el cien por cien del capital sea público no implica una presunción de instrumentalidad y control pleno, como defiende el TJUE, se trataría de un indicio decisivo, sin que constituya en ningún caso un presunción afirmativa del carácter de medio propio. (TJUE, Carbotermo SpA. C-340/04. 11-5-2005).

Pero lo decisivo en el presente análisis es si procede una interpretación analógica y llevar la situación que proclama la ley, que se refiere exclusivamente a las sociedades, al ámbito de las fundaciones y su equivalente en términos económicos, la composición de la dotación fundacional.

En una primera lectura, es sencillo decantarse por la negación de la pretendida interpretación analógica, la ley se refiere exclusivamente al ámbito de las sociedades, obsérvese la forma en que aparece expresado en la ley, "si se trata de sociedades", parece excluir de esta norma a otro tipo de entidades.

Diferente era la redacción de esta cuestión en la antigua Ley de Contratos de las Administraciones Públicas (Real Decreto Legislativo 2/2000), que en el articulo 3.1.1 excluía del ámbito de aplicación de la ley a las encomiendas de gestión que se confieran a entidades y sociedades cuyo capital sea en su totalidad de titularidad pública y sobre las que la Administración que efectúa la encomienda ostente un control análogo al que ejerce sobre sus propios servicios, siempre que estas sociedades y entidades realicen la parte esencial de su actividad con la entidad o entidades que las controlan. Con lo que la excepción afectaba no sólo a las sociedades sino también a otras entidades, incluidas, por qué no, las fundaciones. No obstante el texto de la vigente Ley 30/2007, recoge única y exclusivamente a las sociedades.

La negación de la posibilidad de ser medio propio de las sociedades con participación privada obedece a una premisa expuesta por el TJUE en la sentencia Stadt Halle. C-26/03 de 11-1-2005, en la que afirma que cualquier inversión de capital privado en una empresa obedece a consideraciones propias de los intereses privados, que entraría en conflicto con el interés público que puede hacerse valer en las relaciones entre entidades públicas. Ese interés económico, en principio, quedaría fuera del ámbito de las fundaciones, que por 
propia definición son entidades sin ánimo de lucro, por lo que el actor privado que participe en ellas, puede tener otros intereses, pero nunca los puramente económicos.

Obsérvese también que la realidad de las fundaciones es completamente diferente a las sociedades mercantiles. En estas la participación en el capital social es directamente proporcional al poder de decisión que se ostenta en el ámbito de la sociedad, cosa que no ocurre en las fundaciones, en las que se puede dar el caso que existan patronos que no participen en la dotación fundacional, y el inverso, es decir, que existan aportadores a la dotación que no tengan presencia en el órgano de gobierno, en nuestro caso el patronato. De esto se deduce que aportación a la dotación y control de la sociedad no van de la mano, como ocurre en las sociedades.

En esta línea se muestra la Junta Consultiva de Contratación Administrativa de Cataluña:

Ahora bien, respecto el argumento del Tribunal de Fusticia de las Comunidades Europeas, según el cual toda inversión de capital privado en una empresa obedece a consideraciones características de los intereses privados y persigue objetivos de naturaleza diferente a su objetivo de interés público que persiguen las relaciones entre una entidad adjudicadora y sus propios servicios, siendo posible interpretar, respecto de una fundación, que la presencia de entidades privadas pero sin ánimo de lucro, no alteraría los objetivos de interés público que persiguen en el ente público en que participen mayoritariamente (Informe 4/2007, de 28 de junio, de la Comisión Permanente de la Junta Consultiva de Contratación Administrativa de la Generalitat de Cataluña.)

Por tanto, si bien es cierto que la exigencia de este requisito no plantea dudas por lo que se refiere a sociedades, es posible considerar, en cambio, que en otros tipos de entes, como son las fundaciones u otras entidades sin ánimo de lucro, la exigencia de este requisito requiere algunas matizaciones. El motivo fundamental se basaría en el hecho que la participación minoritaria de estas entidades en consorcios con objetivos y finalidades públicas coincidentes con los que le son propias, no afectaría el principio de la libre circulación de servicio y no alteraría la competencia, motivo este que el TFCE ha utilizado para exigir la concurrencia de los requisitos anteriormente analizados por considerar que una relación jurídica contractual es un contrato interno o in house providing (Informe 9/2009, de 3 de julio, de la Comisión Permanente de la Junta Consultiva de Contratación Administrativa de la Generalitat de Cataluña).

En el ámbito concreto de las fundaciones universitarias es frecuente que la aportación a la dotación fundacional la realice íntegramente la universidad en 
el momento de la constitución de la fundación y que una vez constituida se invite a participar a otras entidades tanto públicas como privadas, esa participación suele articularse por su inclusión en el patronato en calidad de patrono, y puede que ello conlleve una aportación económica a la fundación, aportación económica que no necesariamente debe ir destinada a incrementar la dotación fundacional.

Por tanto, la casuística en las fundaciones es muy diversa, y en ningún caso concuerda con el modelo simplista de las sociedades proclamado en la ley.

Para complicar un análisis hasta ahora sencillo, nos encontramos que en el ámbito del derecho positivo, el legislador andaluz ha realizado una interpretación diferente a la que venimos defendiendo. En concreto la Ley 9/2007, de la Administración de la Junta de Andalucía:

El artículo 52 de la mencionada ley incluye a las fundaciones del sector público andaluz (definidas en los artículos 55 y siguientes de la Ley 10/2005, de Fundaciones de la Comunidad Autónoma de Andalucía) dentro del concepto de entidades instrumentales privadas de la Administración de la Junta de Andalucía, de las que como el resto de entidades instrumentales es predicable el principio de instrumentalidad, en virtud del cual los fines y objetivos que se les asignan específicamente son propios de la Administración de la que dependen.

Por su parte el artículo 106 establece que las fundaciones del sector público tendrán la consideración de medio propio instrumental de la Administración de la Junta de Andalucía o de sus agencias, a los efectos de la ejecución de obras, trabajos, asistencias técnicas y prestación de servicios que se les encomienden, siempre que la aportación constitutiva sea en su totalidad de titularidad pública. Pero afinando más el asunto, la Ley 3/2008, de 23 de diciembre, del Presupuesto de la Comunidad Autónoma de Andalucía para el año 2009, exige además que las entidades instrumentales del sector público andaluz cuyo capital, aportación constitutiva o participación pertenezca integramente a entes de dicho sector. Por lo que, en el caso de que una fundación pueda ser considera medio propio, no solo exige que el cien por cien de la dotación sea pública sino que además provenga de entidades del sector público andaluz, lo que hace mucho más restrictiva y exigente la teoría del "house providing".

Interpretación, como vemos, contraria a la línea general basada en la diferenciación entre sociedades y resto de entidades. Para el legislador andaluz las fundaciones "in house providing" no pueden tener participación privada de ninguna clase y si la tuvieran no podrían en ningún caso ser medio propio ins- 
trumental de la Junta de Andalucía ni, por lo tanto, recibir encomiendas de gestión.

La interpretación dada por el legislador, es la primera interpretación que alcanza grado de ley y, por tanto, de aplicabilidad general, pero eso sí, ceñida al ámbito de los entes instrumentales de la Junta de Andalucía, sin que en ningún caso sea extensible la interpretación a otros ámbitos como el de las universidades, para el que sigue siendo válida la legislación básica estatal en la materia.

Damos por sentado que la participación de entidades de carácter privado en el gobierno o en la dotación de fundaciones universitarias no constituye un impedimento para considerar a éstas como medio propio instrumental de la correspondiente universidad, es fácil deducir que la participación de la referida entidad privada, ha de ser minoritaria, y si son varias, la suma de todas en ningún caso puede suponer la mayoría de la aportación a la dotación, ni de votos en el patronato, pues en este caso estaríamos negando la existencia del control análogo, que sustenta todo el planteamiento expuesto.

Otra presunción iuris et de iure, que en este caso juega a favor de existencia del control análogo, es la indicada en el segundo párrafo del artículo 24.6 se entenderá que los poderes adjudicadores ostentan sobre un ente, organismo o entidad un control análogo al que tienen sobre sus propios servicios si pueden conferirles encomiendas de gestión que sean de ejecución obligatoria para ellos de acuerdo con instrucciones fijadas unilateralmente por el encomendante y cuya retribución se fije por referencia a tarifas aprobadas por la entidad publica de la que dependan.

La LCSP ha utilizado un término puramente administrativista para definir relaciones entre los poderes adjudicadores y sus entes instrumentales, sean estos de naturaleza pública o privada, ya que habla de la encomiendas de gestión, como figura articuladora de la relación.

El régimen jurídico de la encomienda de gestión que se regula en este artículo no será de aplicación cuando la realización de las actividades enumeradas en el apartado primero haya de recaer sobre personas fisicas o jurídicas sujetas a derecho privado, ajustándose entonces, en lo que proceda, a la legislación correspondiente de contratos del Estado, sin que puedan encomendarse a personas o Entidades de esta naturaleza actividades que, según la legislación vigente, hayan de realizarse con sujeción al derecho administrativo (Artículo 15.5, Ley 30/1992).

Pero entendemos que la encomienda de gestión debe ser entendida en es- 
te caso en su acepción más amplia, sin ceñirnos al concepto puramente administrativista del artículo 15 de la Ley 30/1992 del que quedarían claramente excluidas las relaciones entre la administración y sus fundaciones instrumentales privadas. Lo esencial no es el término expresado sino las características que definen ese encargo o prestación:

- Que sean de ejecución obligatoria para la entidad instrumental.

- Que se realicen de acuerdo a instrucciones fijadas unilateralmente por la entidad encomendante.

- Que la retribución por la realización de las mismas se fije por referencia a tarifas aprobadas por la entidad de la que dependan.

Estas son las premisas que debe reunir una determinada actuación encargada por la universidad a su fundación instrumental, independientemente del nombre que se de a al mecanismo a través del cual se realiza el encargo.

\section{$2^{\circ}$. Que realice la parte esencial de su actividad para la uni- versidad}

Esta exigencia constituye el enunciado del principio de instrumentalidad, en virtud del cual las fundaciones son creadas por las universidades y actúan como instrumentos para la consecución de sus fines, realizando la parte esencial de su actividad para las mismas.

El principio de instrumentalidad aparece expresado en numerosas leyes de derecho público como elemento sustancial de las entidades instrumentales de la administración:

El artículo 44.1 de la Ley 6/1997, de 14 de abril, de Organización y Funcionamiento de la Administración General del Estado (LOFAGE) establece respecto de los organismo públicos que se ajustarán al principio de instrumentalidad respecto de los fines y objetivos que tengan específicamente asignados.

Por su parte, el artículo 51 de la Ley 9/2007 de la Administración de la Junta de Andalucía, establece respecto de las entidades instrumentales que se ajustarán al principio de instrumentalidad en virtud del cual los fines y objetivos que se les asignan especificamente son propios de la Administración de la que dependen. 
El artículo 46.1.b) de la Ley 50/2002, de Fundaciones, establece respecto de las fundaciones del sector público estatal que únicamente podrán realizar actividades relacionadas con el ámbito competencial de las entidades del sector público estatal fundadoras, debiendo coadyuvar a la consecución de los fines de las mismas, sin que ello suponga la asunción de sus competencias propias, salvo previsión legal expresa.

El artículo 57.1 de la Ley 10/2005 de Fundaciones de la Comunidad Autónoma de Andalucía, por su parte, establece respecto de las fundaciones del sector público andaluz que podrán realizar únicamente actividades relacionadas con el ámbito competencial de las entidades fundadoras, debiendo contribuir a la consecución de los fines de las mismas, sin que ello suponga la asunción de la titularidad de las competencias de éstas, salvo previsión legal expresa.

Por último, el Artículo 42 de la Ley 5/2010, de 11 de junio, de Autonomía Local de Andalucía, respecto del régimen jurídico de las fundaciones públicas locales, establece que sólo podrán realizar actividades relacionadas con el ámbito competencial de las entidades locales fundadoras, debiendo contribuir a la consecución de los fines de las mismas, sin que ello suponga la asunción de la titularidad de las competencias de estas.

Las fundaciones universitarias, no se pueden encuadrar en ninguna de estas categorías de fundaciones del sector público, estatal, autonómico o local, por tanto no deben considerarse incluidas en ninguna de las categorías apuntadas. Pero, si queremos que sean consideradas medio propio de la universidad deben cumplir con esta exigencia y deben considerarse incluidas dentro del ámbito de este principio, y, por tanto, de su enunciado, por lo que podemos afirmar que las fundaciones universitarias deben realizar la parte esencial de su actividad para la universidad fundadora.

El preámbulo de la Ley 6/2001, Orgánica de Universidades reconoce implícitamente la vigencia de este principio al reconocer a las universidades la competencia para la constitución de fundaciones y otras figuras jurídicas para el desarrollo de sus fines, con lo que se está proclamando abiertamente el principio de instrumentalidad al identificar los fines de la universidad y de las fundaciones $\mathrm{u}$ otras entidades que éstas pudieran crear.

Y ¿qué debemos entender por parte esencial de su actividad?

Sobre este particular también hay jurisprudencia del TJUE

... sólo cabe considerar que la empresa de que se trata realiza lo esencial de su ac- 
tividad con el ente territorial que la controla, cuando la actividad de dicha empresa está destinada principalmente a dicho ente territorial, de modo que el resto de su actividad tiene un carácter meramente marginal.

Para valorar el cumplimiento de las anteriores exigencias se han de tener en cuenta las circunstancias cuantitativas y cualitativas concurrentes en cada caso, y para ello, se ha de tomar en consideración el volumen de negocios realizado por el medio propio por encargo del poder adjudicador, con independencia de si el beneficiario de la actividad es el propio poder adjudicador o los usuarios de los servicios, de modo que es irrelevante quién remunera a la empresa pública (la Administración o los usuarios) y el ámbito territorial de la prestación. (Carbotermo SpA. C-340/04. 11-5-2005. 59).

La citada afirmación del TJUE llevada al ámbito de las fundaciones universitarias, llevaría a afirmar que la mayor parte de su actividad en términos económicos y de empleo de recursos debe ser para la universidad, su participación en contratos con otras entidades y otras fuentes de financiación deben ser marginales o complementarios a la principal que es su relación instrumental de servicios con la universidad.

\section{La condición de medio propio y servicio técnico de las fundaciones universitarias deberá reconocerse expresa- mente por la norma que las cree o por sus estatutos}

Es la exigencia aparentemente más sencilla de cumplir por parte de las fundaciones universitarias, pues depende de un simple acto unilateral de afirmación de la condición de medio propio. Basta con que lo establezcan los estatutos desde un principio o bien, que se lleve a cabo una simple reforma de los mismos. Pero esa afirmación recogida en los estatutos no es baladí, se trata de una afirmación que puede tener consecuencias colaterales que pueden mermar el crecimiento, desarrollo y cumplimiento de los fines de la propia fundación, como veremos más adelante.

La mención expresa de los estatutos no es un mero trámite formal sino de un requisito sustantivo y previo para que la teoría del "house providing" sea efectiva. Así se deduce de las conclusiones del Informe 21/2008, de 27 de noviembre, de la Junta Consultiva de Contratación Administrativa de Andalucía, sobre la legislación aplicable a las encomiendas que la Universidad de Huelva realice a la Corporación de la Universidad de Huelva, S.A.U.:

"En la Corporación de la Universidad de Huelva, S.A.U. concurren las cir- 
cunstancias para considerarla medio propio de la Universidad de Huelva a los efectos previstos en el artículo 24.6 de la LCSP, excepto el reconocimiento expreso de su condición de tal medio que no aparece acreditado ni en la escritura de constitución ni en los estatutos, siéndole aplicable a las encomiendas de gestión que se le confiera el régimen previsto en el artículo 4.1.n) o en el artículo 24 de la LCSP en función de cómo se haya articulado la encomienda".

Teniendo en cuenta lo expuesto hasta este momento, entendemos que las fundaciones universitarias pueden tener la condición de medio propio instrumental de la universidad de la que dependan, y que para que la línea argumental esté completa la universidad debe ser "propietaria" de la mayoría de la fundación, tanto en términos económicos (mayoría de la dotación fundacional) como en términos de gobierno (mayoría de votos en el patronato) cualquier otra configuración pondría en tela de juicio la afirmación del control análogo. Por tanto, la mención que a este respecto realicen los estatutos de la fundación debe constituir la culminación de un proceso de adaptación a todas estas exigencias fácticas.

Difícil argumentación tendría el hecho de que unos estatutos establecieran la condición de medio propio de la fundación respecto de más de una entidad, ya que una y solo una de esas entidades puede ser mayoritaria.

\section{La condición de medio propio determinará para ellas la imposibilidad de participar en licitaciones públicas con- vocadas por los poderes adjudicadores de los que sean medios propios}

Está claro según esta cuarta condición de la teoría del "house providing" que una fundación universitaria no puede bajo ningún concepto participar en una licitación convocada por la universidad de la que dependa. El hilo argumental de esta teoría caería como fichas de dominó si esto no fuera así.

La única salvedad (que no constituye ninguna salvedad sino una nueva forma de manifestarse la teoría del medio propio) sería que no concurriera ninguna oferta a la licitación convocada, en este caso y sólo en este caso, la universidad podría encargarle a la fundación la ejecución de la prestación objeto de las mismas.

Descartada pues la posibilidad de presentarse a licitaciones convocadas por la universidad de la que depende, habría que plantearse si las fundaciones 
universitarias, en su condición de fundaciones públicas, podrían concurrir a licitaciones convocadas por otros poderes adjudicadores, incluidas otras universidades diferentes a aquélla de la cual dependen.

La Directiva 2004/18/CE, admite implícitamente la posibilidad que entes del sector público participen como licitadores en procedimientos de adjudicación:

Los Estados miembros deben velar por que la participación en un procedimiento de adjudicación de contrato público de un licitador que sea un organismo de derecho público no cause distorsión de la competencia con respecto a licitadores privados (Considerando 4)

Recordemos que en la citada Directiva (artículo 9) el concepto de organismo de Derecho público es diferente al concepto jurídico español, ya que para aquélla es cualquier organismo en que concurren las siguientes circunstancias:

a) Que haya sido creado especificamente para satisfacer necesidades de interés general que no tengan carácter industrial o mercantil,

b) Que esté dotado de personalidad jurídica y

c) Que su actividad esté mayoritariamente financiada por el Estado, los entes territoriales u otros organismos de Derecho público, o bien cuya gestión se halle sometida a un control por parte de estos últimos, o bien cuyo órgano de administración, de dirección o de vigilancia esté compuesto por miembros de los cuales más de la mitad sean nombrados por el Estado, los entes territoriales u otros organismos de Derecho público.

Las características expuestas, coinciden con el concepto legal de poder adjudicador, de la legislación española. Por tanto, las fundaciones universitarias son organismo de derecho público, según el concepto comunitario, y, por tanto, podrían participar en procedimientos de adjudicación, con el único límite que la propia directiva impone a los estados miembro con carácter general y de manera muy ambigua, que no cause distorsión de la competencia con respecto a licitadores privados, que en principio no debe suponer ninguna restricción apriorística de la posibilidad de participación.

La posibilidad de celebrar contratos con la administración y, como consecuencia, la posibilidad de participar en licitaciones públicas aparece expresamente reconocida para las universidades y/o sus entidades gestoras de la in- 
vestigación en el artículo 83.1 de la Ley 6/2001, de 21 de diciembre Orgánica de Universidades:

Los grupos de investigación reconocidos por la Universidad, los Departamentos y los Institutos Universitarios de Investigación, y su profesorado a través de los mismos o de los órganos, centros, fundaciones o estructuras organizativas similares de la Universidad dedicados a la canalización de las iniciativas investigadoras del profesorado y a la transferencia de los resultados de la investigación, podrán celebrar contratos con personas, Universidades o entidades públicas y privadas para la realización de trabajos de carácter científico, técnico o artístico, asi como para el desarrollo de enseñanzas de especialización o actividades especificas de formación.

Tal previsión de la LOU aparece en la propia LCSP, en cuya Disposición adicional novena establece

No será exigible la clasificación a las Universidades Publicas para ser adjudicatarias de contratos en los supuestos a que se refiere el apartado 1 del articulo 83 de la Ley Orgánica 6/2001, de 21 de diciembre, de Universidades. Exención ésta que entendemos puede hacerse extensible por analogía a las fundaciones universitarias cuando actúen como entidades canalizadoras de las iniciativas investigadoras del profesorado en licitaciones públicas.

Por tanto, las fundaciones universitarias al igual que las propias universidades están legalmente habilitadas para participar en licitaciones convocadas por otros poderes adjudicadores, lógicamente el ámbito de participación se ciñe a aquellos trabajos en los que las fundaciones y la propia universidad pueden resultar competitivos que no son otros que los trabajos de carácter científico, técnico o artístico, así como para el desarrollo de enseñanzas de especialización o actividades específicas de formación.

Pero aquí entran en juego los límites a los que ya nos hemos referido:

- Que no se altere la libre competencia

- Que esos contratos en términos de volumen de negocio tengan carácter residual.

El primero de estos límites emana directamente de la Directiva 2004/18/CE, como ya hemos apuntado y constituye un límite muy general y casi obligado en el discurso corriente de las instituciones europeas, para las que la defensa del libre mercado y la libre competencia constituyen su razón de ser. 
Evidentemente esta expresión contenida en el considerando de la Directiva, no proyecta ninguna exigencia concreta sobre la entidad pública que participe en la licitación, no podemos afirmar de manera categórica cuando puede concurrir esa distorsión de la libre competencia que rompa la igualdad con los licitadores privados, habrá que estar a cada caso concreto.

Lo que parece deducirse es que existe una obligación de los poderes adjudicadores de velar por que las reglas de la libre competencia jueguen en todo momento y son ellos los llamados a advertir la presencia de elementos que distorsionen el juego, como puede ser la presentación por parte de una fundación universitaria de un precio muy inferior al presupuesto de licitación porque una buena parte de los gastos derivados del servicio que se aspira a prestar ya están cubiertos por los presupuestos de la universidad o por otras vías, procedente siempre de fondos públicos, y no se repercuten como gasto del contrato. Entendemos que en estos casos debe intervenir la advertencia establecida en la Directiva y el poder adjudicador convocante debe excluir a la entidad licitadora por distorsionar las reglas de la libre competencia.

Si no fuera así, entendemos que sería un buen argumento en manos de los licitadores privados para interponer recurso especial en materia de contratación contra el acuerdo de adjudicación que pudiera adoptarse.

El segundo de los límites es consecuencia directa de uno de los elementos que forman el concepto de medio propio instrumental de las fundaciones respecto de las universidades, que como hemos visto han de realizar la parte esencial de la actividad para la entidad pública de la que dependen. Si esa debe ser la parte esencial de su actividad, en términos de volumen de negocio, lógicamente el resto de actuaciones que lleve a cabo con terceros, debe tener carácter residual. Si no fuera así no podría ser considerada medio propio instrumental y no podría, por tanto, realizar contratos y actuaciones para la universidad sin someterse a las formalidades exigidas en la LCSP.

Por último, analizamos en este punto la Disposición adicional décima de la LCSP, que aparece bajo la rúbrica "Exención de requisitos para los Organismos Públicos de Investigación en cuanto adjudicatarios de contratos".

1. Las Agencias Estatales, los Organismos Públicos de Investigación y organismos similares de las Comunidades Autónomas no necesitarán estar clasificados ni acreditar su solvencia económica y financiera y la solvencia técnica para ser adjudicatarios de contratos del sector público. 
2. Estas entidades estarán igualmente exentas de constituir garantías, en los casos en que sean exigibles.

¿Pueden considerarse las fundaciones universitarias dentro del ámbito de aplicación de esta excepción? La respuesta es clara y contundentemente negativa, esa excepción no se puede aplicar a las fundaciones ni tampoco a las propias universidades, ni por entenderla incluidas en el ámbito subjetivo ni por analogía. La excepción se refiere exclusivamente a los Organismos Públicos de Investigación, con mayúsculas, éstos organismos, que incluso tienen su propio acrónimo, OPI, se encuentran enumerados en el artículo 13 de la Ley 13/1986, de 14 de abril, de Fomento y Coordinación de la Investigación Científica y Técnica (FCIC), esta enumeración constituye un numerus clausus, en el sentido que son estos organismo y no otros:

El Consejo Superior de Investigaciones Científicas (CSIC,)

El Centro de Investigaciones Energéticas, Medioambientales y Tecnológicas (CIEMAT)

El Instituto Geológico y Minero (IGME), el Instituto Nacional de Técnica Aeroespacial (INTA).

El Instituto Nacional de Investigación y Tecnología Agraria y Alimentaria (INIA).

El Instituto Español de Oceanografía (IEO)

El Instituto de Salud Carlos III

Queda claro que ni la universidad ni sus fundaciones dependientes pueden ser considerados Organismos Públicos de Investigación, pero la ley amplia esta excepción a los organismos similares de las Comunidades Autónomas, donde entendemos que tampoco se pueden encuadrar. Muy ilustrativo resulta en este sentido el Informe 7/2008, de 10 de julio, de la Junta Consultiva de Contratación Administrativa de la Comunidad Autónoma de Madrid, que analiza precisamente el caso de una fundación del sector público dedicada a la investigación biomédica:

Para responder a la primera cuestión planteada relativa a posibilidad de asimilar la Fundación a un organismo público de investigación estatal, se debe analizar la regulación de ambos tipos de entidades, teniendo en cuenta que el articulo 4 q) de la LCSP recoge como 
negocios excluidos del ámbito de la Ley los contratos de servicios y suministros celebrados por organismos públicos de investigación estatales y los organismos similares de Comunidades Autónomas que tengan por objeto prestaciones o productos necesarios para la ejecución de proyectos.

El artículo 13 de la Ley 13/1986, de 14 de abril, de Fomento y Coordinación de la Investigación Científica y Técnica (FCIC), relaciona los organismos públicos de investigación del Estado y dispone que se regirán por la citada Ley, por su legislación especial y por la Ley 6/1997, de 14 de abril, de Organización y Funcionamiento de la Administración General del Estado (LOFAGE). La LOFAGE, en su articulo 43, define los organismos públicos como aquellos creados bajo la dependencia y vinculación de la Administración General del Estado para realizar actividades que justifiquen su organización y desarrollo en régimen de descentralización funcional, tienen personalidad jurídica diferenciada, patrimonio y tesorería propia, autonomía de gestión y en los términos de la Ley y dentro de su esfera de competencia, les corresponden las potestades administrativas precisas para el cumplimiento de sus fines en los términos que prevean sus estatutos, salvo la potestad expropiatoria. En la clasificación de los organismos públicos que realiza el citado artículo 43, se encuentran los organismos autónomos y las entidades públicas empresariales.

En el artículo 15.2 de la FCIC se diferencia a estos organismos de investigación de las Fundaciones al disponer que aquéllos podrán suscribir convenios de colaboración con universidades, con fundaciones o con instituciones sin ánimo de lucro, tanto nacionales como extranjeras, para la ejecución y desarrollo de las actividades, entre las que cita: proyectos de investigación científica, desarrollo e innovación tecnológica, transferencia de conocimientos y de resultados científicos, creación, gestión o financiación de centros o unidades de investigación.

\section{La fundación universitaria debe actuar conforme a lo dis- puesto en la ley para llevar a cabo las encomiendas de gestión}

Como hemos tenido oportunidad de analizar, las fundaciones universitarias pueden tener la consideración de medio propio instrumental de la universidad que las crea, pero independientemente de tal afirmación parece claro que en el ámbito de la contratación de las obras, bienes y servicios que realice, tiene la categoría de poder adjudicador, por lo que para finalizar este documento traemos a colación la exigencia recogida en el artículo 4.1.m de la LCSP

... los contratos que deban celebrarse por las entidades que tengan la consideración de medio propio y servicio técnico para la realización de las prestaciones objeto del encargo 
quedaran sometidos a esta Ley, en los términos que sean procedentes de acuerdo con la naturaleza de la entidad que los celebre y el tipo y cuantía de los mismos...

En el caso analizado se trataría del régimen de contratación que la ley prevé para los poderes adjudicadores que no tienen el carácter de administración pública. Un complejo sistema de contratación cuyo análisis consideramos queda fuera del ámbito y las pretensiones del presente artículo. 